\title{
BMJ Open Joint impact of dementia and frailty on healthcare utilisation and outcomes: a retrospective cohort study of long-stay home care recipients
}

\author{
Colleen J Maxwell, ${ }^{\oplus 1,2}$ Luke Mondor, ${ }^{2,3}$ David B Hogan, ${ }^{4}$ Michael A Campitelli, ${ }^{2}$ \\ Susan E Bronskill, ${ }^{2,5}$ Dallas P Seitz, ${ }^{6,7}$ Walter P Wodchis ${ }^{\circledR, 8}$
}

To cite: Maxwell CJ, Mondor L, Hogan DB, et al. Joint impact of dementia and frailty on healthcare utilisation and outcomes: a retrospective cohort study of long-stay home care recipients. BMJ Open 2019;9:e029523. doi:10.1136/ bmjopen-2019-029523

\section{- Prepublication history and} additional material for this paper are available online. To view these files, please visit the journal online (http://dx.doi. org/10.1136/bmjopen-2019029523).

Received 30 January 2019 Revised 15 April 2019 Accepted 30 May 2019
Check for updates

(C) Author(s) (or their employer(s)) 2019. Re-use permitted under CC BY-NC. No commercial re-use. See rights and permissions. Published by BMJ.

For numbered affiliations see end of article.

Correspondence to

Colleen J Maxwell;

colleen.maxwell@uwaterloo.ca

\section{ABSTRACT}

Objectives To examine the associations between

dementia and 1-year health outcomes (urgent

hospitalisation, long-term care (LTC) admission,

mortality) among long-stay home care recipients and the

extent to which these associations vary by clients' frailty level.

Design A retrospective cohort study using linked clinical and health administrative databases.

Setting Home care in Ontario, Canada.

Participants Long-stay ( $\geq 60$ days) care clients

$(n=153125)$ aged $\geq 50$ years assessed between April 2014 and March 2015.

Main outcome measures Dementia was ascertained with a validated administrative data algorithm and frailty with a 66 -item frailty index (FI) based on a previously validated FI derived from the clinical assessment. We examined associations between dementia, $\mathrm{Fl}$ and their interactions, with 1-year outcomes using multivariable Fine-Gray competing risk (urgent hospitalisation and LTC admission) and Cox proportional hazards (mortality) models.

Results Clients with dementia (vs without) were older (mean $\pm S D, 83.3 \pm 7.9$ vs $78.9 \pm 11.3$ years, $p<0.001$ ) and more likely to be frail ( $30.3 \%$ vs $24.2 \%, p<0.001)$. In models adjusted for $\mathrm{Fl}$ (as a continuous variable) and other confounders, clients with dementia showed a lower incidence of urgent hospitalisation (adjusted subdistribution $\mathrm{HR}(\mathrm{sHR})=0.84,95 \% \mathrm{Cl}: 0.83$ to 0.86 ) and mortality rate (adjusted $\mathrm{HR}=0.87,95 \% \mathrm{Cl}: 0.84$ to 0.89 ) but higher incidence of LTC admission (adjusted $\mathrm{SHR}=2.60$, $95 \%$ Cl: 2.53 to 2.67). The impact of dementia on LTC admission and mortality was significantly modified by clients' FI ( $p<0.001$ interaction terms), showing a lower magnitude of association (ie, attenuated positive (for LTC admission) and negative (for mortality) association) with increasing frailty.

Conclusions The strength of associations between dementia and LTC admission and death (but not urgent hospitalisation) among home care recipients was significantly modified by their frailty status. Understanding the public health impact of dementia requires consideration of frailty levels among older populations, including those with and without dementia and varying degrees of multimorbidity.

\section{Strengths and limitations of this study}

- This population-based long-stay home care study included a large sample size and employed robust statistical modelling techniques to explore relevant interactions and to account for competing risks over follow-up.

- Both exposures of interest (dementia and frailty) were based on previously validated measures for older care recipients in Ontario.

- The availability of linked clinical and health administrative databases allowed for an investigation of the impact of a comprehensive, multidomain frailty index on dementia-outcome associations of interest.

- Findings from this study may not be generalisable to community-residing older adults not currently receiving home care services on a long-stay basis.

- Data regarding other covariates (eg, support services received) and health outcomes (eg, functional and/or cognitive decline, quality of life) of interest to home care clients were not available for this cohort and should be explored in future research.

\section{BACKGROUND}

An estimated 500000 Canadians currently live with dementia and this number is expected to double over the next $10-15$ years. ${ }^{1}$ Though increasing functional impairment and behavioural challenges often lead to institutionalisation, many with dementia reside in the community with substantial support provided by family, friends and formal home care services. ${ }^{23}$ Beyond the implications for the health and well-being of those living with or affected by dementia, projected increases in dementia prevalence raise concerns about the ability of the healthcare system to deal with anticipated demand and costs. ${ }^{14}$

Previous work, largely from the USA, has demonstrated elevated healthcare utilisation and expenditures for community-dwelling older adults with dementia relative to matched comparison groups. ${ }^{56}$ This includes an increased 
likelihood for hospitalisation, ${ }^{7-10}$ emergency department visits, ${ }^{79}$ and long-term care (LTC) placement. ${ }^{11} 12$ These utilisation patterns are important from a public payer perspective but may also highlight possible inadequacies in the availability and/or effectiveness of community-based care for persons with dementia. ${ }^{12}$ Many of the resultant transitions in care, especially hospitalisations, are associated with worse outcomes for those with dementia, ${ }^{13}$ and may be potentially avoidable with timely and adequate care in the community setting. ${ }^{6-814}$ Recent healthcare reforms in Canada and elsewhere have called for an expansion of publicly funded home and community-based care ${ }^{1516}$ with the aim of potentially reducing costly acute and LTC admissions among vulnerable older adults. Consequently, there is considerable value in understanding patterns of healthcare utilisation among older home care recipients, especially for persons with dementia and/or other indices of heightened risk or vulnerability. ${ }^{2317-19}$

Population-based reports on the impact of dementia on health outcomes and healthcare use among vulnerable older adults are relatively scarce in Canada, ${ }^{4}$ with the exception of a few recent studies on dementia in the context of multimorbidity only, ${ }^{320}$ including previous work by our team. ${ }^{3}$ Notably absent are studies examining the joint impact of dementia and frailty on healthcare outcomes in community-dwelling older adults, ${ }^{21}{ }^{22}$ including those receiving care in the home. Frailty, defined as an increased vulnerability to stressors arising from multisystem dysfunction and subsequent loss of homoeostatic reserve and resiliency, ${ }^{23}$ is an important predictor of care transitions among older populations,${ }^{24}{ }^{25}$ though its predictive value in dementia is less clear. ${ }^{26}{ }^{27}$ Emerging data support a bidirectional relationship between frailty and dementia ${ }^{2829}$ with both becoming more common with increasing age. ${ }^{4124}$ As frailty level may reflect dementia severity or stage as well as overall vulnerability, it is an important consideration in understanding the health system implications of dementia prevalence trends.

To inform current and future regional and national dementia strategies ${ }^{30}$ and related policy and resource planning decisions regarding home and community-based services for this vulnerable population, we sought to (1) investigate the relative effect of dementia on the incidence of urgent (non-elective) hospitalisation and LTC admission and rate of death over 1 year among a current cohort of community-dwelling home care recipients in Ontario, and (2) explore variation in these associations by client frailty. In doing so, we provide important baseline empirical data to assist with the prioritisation and evaluation of novel client and system-level interventions to improve the healthcare and outcomes of vulnerable persons with and without dementia.

\section{METHODS}

\section{Study design and setting}

We conducted a retrospective cohort study of long-stay home care clients in Ontario from April 2014 to March 2016 using linked health administrative and clinical databases. During this period, Ontario's population included over 13.5 million residents with approximately 5 million aged 50 years and older. Most are covered by a universal, publicly funded health insurance programme for all necessary medical and emergency care services. Included are costs for hospital and physician services and prescription drugs for those aged 65 years and older or on social assistance or receiving services under the home care programme. Referrals for publicly funded home care may be made by healthcare providers, institutions, clients and/or their family and potential clients are assessed for eligibility and level of care by regional case managers. Services may include homemaking, transportation, personal care, nursing care, end-of-life care, physiotherapy, occupational and speech-language therapy and can vary by type and amount across health regions. ${ }^{15}$ Home care is provided on either a short-stay (ie, services provided for $<60$ days (eg, to aid in recovery postsurgery or injury)) or long-stay (ie, clients requiring services in the home for $\geq 60$ days in a single episode) basis. For all long-stay clients (approximately $40 \%$ of all home care clients), ${ }^{31}$ the province has mandated the administration of the Resident Assessment Instrument for Home Care (RAI-HC) on admission and at regular ( 6 month) intervals. The RAI-HC is completed by trained staff and provides standardised data on clients' sociodemographic characteristics, health conditions, physical and cognitive status, behaviours and service use. ${ }^{32}$

RAI-HC data were linked with several provincial administrative databases using unique encoded identifiers and analysed at ICES. These included the Continuing Care Reporting System for Long-Term Care (CCRS-LTC), Canadian Institute for Health Information's Discharge Abstract database (DAD), Ontario Health Insurance Plan (OHIP) database, Ontario Drug Benefit (ODB) database and Registered Persons Database (RPDB).

The use of data in this project was authorised under section 45 of Ontario's Personal Health Information Protection Act, which does not require review by a Research Ethics Board. Informed consent from participants was not required because we used health information routinely collected in Ontario and held in health administrative databases. The study is reported as per RECORD guidelines (online supplementary S1 table).

\section{Study cohort}

All RAI-HC assessments dated between 1 April 2014 and 31 March 2015 among clients aged 50-105 years $(\mathrm{n}=250987)$ were identified. Records were excluded for data quality issues $(n=609)$ and for those ineligible to receive healthcare services or who resided outside the province $(n=230)$. Given our interest in community-based home care clients, we excluded records for those who had resided in LTC $(\mathrm{n}=8816)$ or had received designated palliative care $(n=14003)$, or only case management $(\mathrm{n}=5775)$ in the year prior to RAI-HC assessment. We excluded clients receiving palliative home care as they represent a unique subgroup with different objectives of 
care and drivers of healthcare utilisation with their own policy and practice implications. ${ }^{33}$ For those with multiple RAI-HC assessments, only the first assessment in the study period was examined (index assessment, $\mathrm{n}=160209$ ). We excluded those in hospital at the time of this assessment $(\mathrm{n}=7084)$, resulting in a final sample of 153125 clients.

\section{Patient and public involvement}

Patients were not involved in the design or conduct of this study.

\section{Dementia and frailty}

Presence of a dementia diagnosis prior to the index assessment was ascertained using a validated algorithm based on the presence of a dementia-related hospitalisation code (DAD), or three physician claims for dementia within a 2-year period each separated by 30 days (OHIP) or a prescription filled for a cholinesterase inhibitor (ODB).$^{34}$

Baseline frailty was defined using a validated frailty index (FI), calculated as the proportion of accumulated to potential health deficits based on 72 variables derived from the index RAI-HC. ${ }^{24}{ }^{25}$ Given our focus on both dementia and frailty as predictors, we excluded dementia diagnoses and cognitive items from the original FI, an approach consistent with that employed by other researchers, ${ }^{35}$ resulting in a 66 -item FI. This FI was examined as a continuous variable, with higher values indicative of greater frailty. In sensitivity analyses, a categorical FI was examined with robust $(\mathrm{FI}<0.2)$, prefrail (FI 0.2-0.3) and frail (FI >0.3) clients identified based on previously defined thresholds. ${ }^{24}$

\section{Covariates}

Client age (at index assessment) and sex were identified from the RPDB, and neighbourhood-level income quintile and rural residence (ie, community with $<10000$ individuals) from the 2006 Statistics Canada census. Marital status was derived from the index RAI-HC. Multimorbidity was based on a count of 16 high-impact chronic conditions (exclusive of dementia) using common case ascertainment algorithms for DAD and OHIP databases. Additional details regarding these conditions and codes are provided in online supplementary S2 table and elsewhere. ${ }^{336}$ Multimorbidity was coded as zero or one, two, three, four, five or six-plus conditions.

\section{Outcomes}

We determined the time (in days) to first urgent hospitalisation (DAD data), first LTC admission (CCRS-LTC data) and death (RPDB data) during the 1-year period following clients' index assessment. Of note, 92\% of first hospital admissions were urgent (ie, non-elective or unplanned).

\section{Statistical analyses}

Descriptive statistics were calculated for baseline characteristics (including frailty) and key outcomes by dementia status, using $\chi^{2}$ tests for categorical variables and one-way analysis of variance for continuous variables.

We modelled associations between dementia, frailty and 1-year outcomes using Fine-Gray competing risk models for urgent hospitalisation (accounting for death and LTC admission) $)^{3}$ and LTC admission (accounting for death) and Cox proportional hazards models for mortality. ${ }^{37}$ Associations are reported as either subdistribution-HRs (sHR, Fine-Gray models) or HRs (Cox models) with corresponding 95\% CIs. For clients where no event was observed, follow-up time was censored at 1 year after the index assessment. For interpretation, continuous FI estimates are expressed per 0.1-unit increase, which equates to $6-7$ additional deficits.

Initial models assessed the separate associations of dementia and frailty with outcomes, adjusting for age and sex. Full multivariable models included dementia and frailty adjusting for age, sex, marital status, income quintile, rural/urban residence and multimorbidity, consistent with previous work. ${ }^{324}$ A two-way dementia-frailty interaction was then added to this model and statistical significance of the regression term assessed. From these models, we estimated the sHR or HR and corresponding CI for dementia (yes vs no) across the FI continuum. To assist with interpretation, we report the estimated associations of dementia with outcomes at the 25th and 75th percentiles of the FI distribution in the study population (FI=0.177 and 0.303 , respectively).

In sensitivity analyses (ie, categorical FI variable), the significance of dementia-frailty interaction terms for all outcomes were examined with Wald tests, with resulting coefficients plotted for visual representation. Coefficients represent sHR or HR for each dementia-frailty group relative to a reference group of robust clients without dementia (considered the lowest risk group for comparative purposes).

Observations with missing data $(<0.4 \%$ of cohort) were excluded from all analyses. All statistical analyses were conducted using Stata/MP V.15.

\section{RESULTS}

The mean age of the sample was $80.1( \pm 10.7)$ years, $65 \%$ were women, almost half were widowed and the majority $(87 \%)$ resided in an urban setting (table 1). Twenty-seven percent $(n=40956)$ had a dementia diagnosis. High levels of multimorbidity were evident. The most prevalent were hypertension $(83.6 \%)$, osteoarthritis $(66.3 \%)$, diabetes $(40.8 \%)$, coronary syndrome $(33.9 \%)$ and congestive heart failure $(26.8 \%)$ (online supplementary S3 table). Clients' mean FI was $0.24( \pm 0.09)$ and $26 \%$ were categorised as frail (with $40 \%$ prefrail and $34 \%$ robust). Clients with dementia (vs without) were significantly more likely to be older, male and to have lower levels of multimorbidity but a higher mean FI, with a greater proportion categorised as frail (30.3\% vs $24.2 \%$ ) (online supplementary S1 figure). 
Table 1 Baseline characteristics and 1-year health outcomes of long-stay home care clients aged 50+ years in Ontario (April 2014 to March 2015), by the presence of dementia

\begin{tabular}{|c|c|c|c|}
\hline & & Dementia diagn & \\
\hline & $\begin{array}{l}\text { Overall sample } \\
n=153125\end{array}$ & $\begin{array}{l}\text { No } \\
n=112169\end{array}$ & $\begin{array}{l}\text { Yes } \\
n=40956\end{array}$ \\
\hline Age (years) & & & \\
\hline Mean \pm SD & $80.08 \pm 10.65$ & $78.92 \pm 11.28$ & $83.27 \pm 7.85$ \\
\hline Median (IQR) & $82(74-88)$ & $81(71-88)$ & 84 (79-89) \\
\hline Female sex & $99040(64.7 \%)$ & $73133(65.2 \%)$ & 25907 (63.3\%) \\
\hline Marital status & & & \\
\hline Married & $58389(38.1 \%)$ & $41127(36.7 \%)$ & $17262(42.1 \%)$ \\
\hline Widowed & $68353(44.6 \%)$ & $49151(43.8 \%)$ & $19202(46.9 \%)$ \\
\hline Separated/divorced & $14771(9.6 \%)$ & $12131(10.8 \%)$ & $2640(6.4 \%)$ \\
\hline Never married/other & $11612(7.6 \%)$ & $9760(8.7 \%)$ & $1852(4.5 \%)$ \\
\hline Rural-urban residence $†$ & & & \\
\hline Urban & $133619(87.3 \%)$ & $97160(86.6 \%)$ & 36459 (89.0\%) \\
\hline Rural & $19502(12.7 \%)$ & $15007(13.4 \%)$ & 4495 (11.0\%) \\
\hline Income quintile† & & & \\
\hline 1 (low) & $36889(24.1 \%)$ & $28642(25.5 \%)$ & $8247(20.1 \%)$ \\
\hline 2 & $32812(21.4 \%)$ & $24444(21.8 \%)$ & $8368(20.4 \%)$ \\
\hline 3 & $29656(19.4 \%)$ & $21503(19.2 \%)$ & $8153(19.9 \%)$ \\
\hline 4 & $28217(18.4 \%)$ & $19943(17.8 \%)$ & $8274(20.2 \%)$ \\
\hline 5 (high) & $24963(16.3 \%)$ & $17193(15.3 \%)$ & $7770(19.0 \%)$ \\
\hline Number of chronic conditions & & & \\
\hline $0-1$ & $12437(8.1 \%)$ & $8312(7.4 \%)$ & $4125(10.1 \%)$ \\
\hline 2 & $20112(13.1 \%)$ & $13805(12.3 \%)$ & $6307(15.4 \%)$ \\
\hline 3 & 28867 (18.9\%) & $20560(18.3 \%)$ & 8307 (20.3\%) \\
\hline 4 & 29459 (19.2\%) & 21660 (19.3\%) & $7799(19.0 \%)$ \\
\hline 5 & $24422(15.9 \%)$ & $18485(16.5 \%)$ & 5937 (14.5\%) \\
\hline $6+$ & $37828(24.7 \%)$ & $29347(26.2 \%)$ & $8481(20.7 \%)$ \\
\hline Frailty index (modified) & & & \\
\hline Mean \pm SD & $0.24 \pm 0.09$ & $0.24 \pm 0.09$ & $0.25 \pm 0.10$ \\
\hline Median (IQR) & $0.23(0.18-0.30)$ & $0.23(0.17-0.30)$ & $0.25(0.18-0.32)$ \\
\hline Robust & $52113(34.0 \%)$ & $39214(35.0 \%)$ & $12899(31.5 \%)$ \\
\hline Prefrail & $61450(40.1 \%)$ & $45788(40.8 \%)$ & $15662(38.2 \%)$ \\
\hline Frail & $39562(25.8 \%)$ & $27167(24.2 \%)$ & $12395(30.3 \%)$ \\
\hline Outcomes over follow-up & & & \\
\hline Died & $22439(14.7 \%)$ & $16334(14.6 \%)$ & $6105(14.9 \%)$ \\
\hline Admitted to LTC & $24704(16.1 \%)$ & $12413(11.1 \%)$ & 12291 (30.0\%) \\
\hline Urgent hospital admission & $58551(38.2 \%)$ & $43504(38.8 \%)$ & 15047 (36.7\%) \\
\hline
\end{tabular}

${ }^{*}$ All differences are statistically significant at $p<0.001$ except for mortality outcome $(p=0.092)$.

†Less than $0.4 \%$ of the cohort with missing data for one or both of these covariates.

LTC, long-term care.

Over the 1 year, a greater proportion of clients with dementia were admitted to LTC $(30.0 \%$ vs $11.1 \%)$, while slightly fewer had an urgent hospitalisation $(36.7 \%$ vs $38.8 \%$ ). The distribution of the most common causes of all urgent hospitalisations by dementia status are shown in supplementary S4 table. Crude mortality did not vary significantly by dementia status ( $15 \%$ for both groups).

In age-sex and fully adjusted models, the incidence of urgent hospitalisation was significantly lower among clients with dementia and higher for those with greater 
Table 2 Estimated associations $†$ between dementia, frailty (and dementia-frailty interaction) and 1-year health outcomes, among long-stay home care clients aged $50+$ years in Ontario

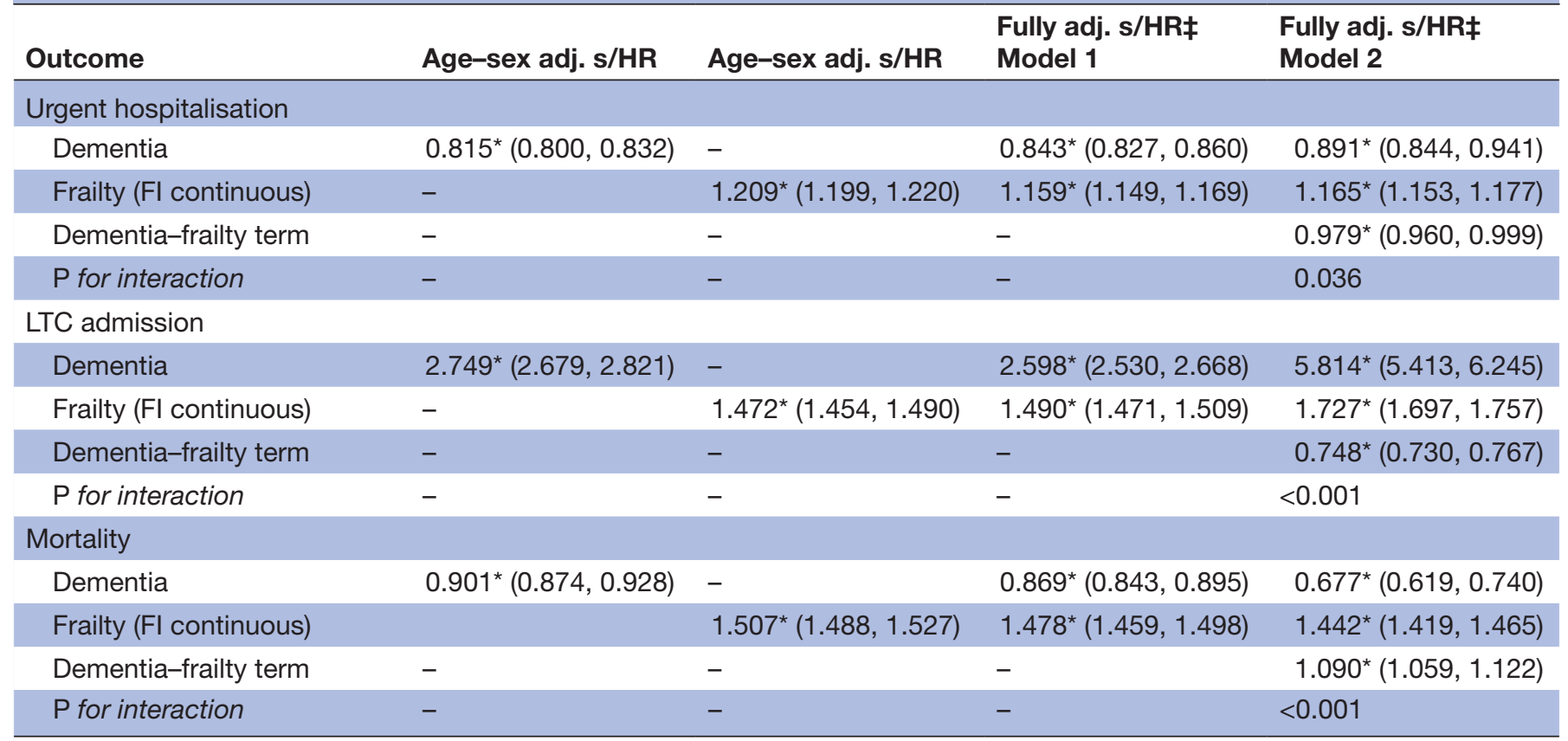

${ }^{*} \mathrm{P}<0.05$.

†For urgent hospitalisation and LTC admission, estimates are subdistribution HRs and corresponding 95\% Cls from Fine-Gray model; for mortality, estimates are HRs and corresponding 95\% Cls from Cox proportional hazards regression model.

$\ddagger$ Models adjusted for age, sex, marital status, rurality, income quintile and multimorbidity count; Model 2 additionally includes dementia-frailty interaction term.

FI, frailty index; LTC, long-term care.

frailty (table 2). The dementia-FI interaction term was modestly significant $(\mathrm{p}=0.036)$ and suggested that the lower incidence of urgent hospitalisation for dementia was slightly more pronounced with increasing frailty (figure 1A). For example, the estimated sHR for urgent hospitalisation associated with dementia at the 25th and 75th percentiles of FI was 0.86 (CI: 0.84 to 0.88 ) and 0.84 (CI: 0.82 to 0.86 ), respectively.

In age-sex and fully adjusted models, both dementia and higher frailty levels were significantly associated with a higher incidence of LTC admission. The dementia-FI interaction term was significant $(p<0.001$, table 2$)$, and showed that the relative magnitude of the increased incidence of LTC admission associated with dementia was lower with increasing frailty (figure 1B). The estimated sHR for LTC admission among those with dementia (vs without) at the 25th and 75th percentiles of FI was 3.48 (CI: 3.36-3.61) and 2.42 (CI: 2.35-2.48), respectively.

The rate of mortality was significantly lower for clients with dementia and higher for those with greater frailty in both age-sex and fully adjusted models. The dementiaFI interaction term was significant $(p<0.001$, table 2$)$ and indicated that the lower mortality rate associated with dementia was attenuated with increasing frailty (figure 1C). The estimated HR for death among clients with dementia (vs without) at the 25th and 75th percentiles of FI was 0.79 (CI: 0.76-0.83) and 0.88 (CI: 0.850.91 ), respectively. At FI levels beyond 0.5 (ie, the most frail $1 \%$ ), clients with dementia showed an increased mortality rate.

\section{Sensitivity analyses}

Incorporating a three-level categorical FI variable (to define robust, prefrail and frail groups) into the models for each outcome produced comparable findings, except that the dementia-FI interaction term was no longer statistically significant for urgent hospitalisation $(\mathrm{p}=0.124$; online supplementary S5 table and figure $2 \mathrm{~A}-\mathrm{C}$ ). Cumulative incidence plots illustrating the dementia-categorical FI associations with each outcome are presented in figure $3 \mathrm{~A}-\mathrm{C}$ (with 1-year estimates shown in online supplementary $\mathrm{S} 6$ table). The latter figures illustrate the magnitude of absolute risk (percentage estimates) for each of our three outcomes across comparison groups that vary in dementia and (categorical) frailty status.

\section{DISCUSSION}

In this population-based study of primarily urban-dwelling older long-stay home care clients in Ontario, just over one quarter had dementia with a similar proportion categorised as frail. Clients with dementia (vs without) were older and more likely to be frail ( $30 \%$ vs $24 \%$ ) but showed lower levels of multimorbidity. Both groups showed meaningful variation in frailty status with close to a third being robust. In adjusted analyses accounting for relevant 
A) Urgent Hospitalization

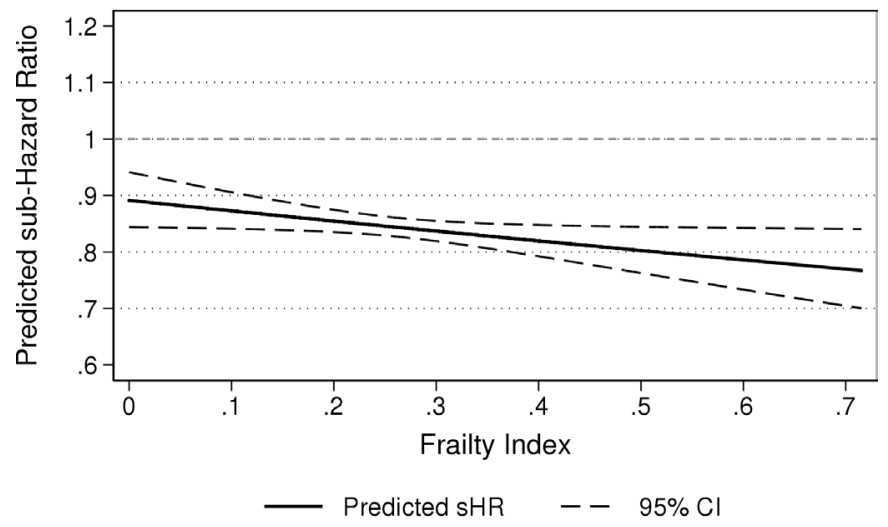

B) Long-Term Care Placement

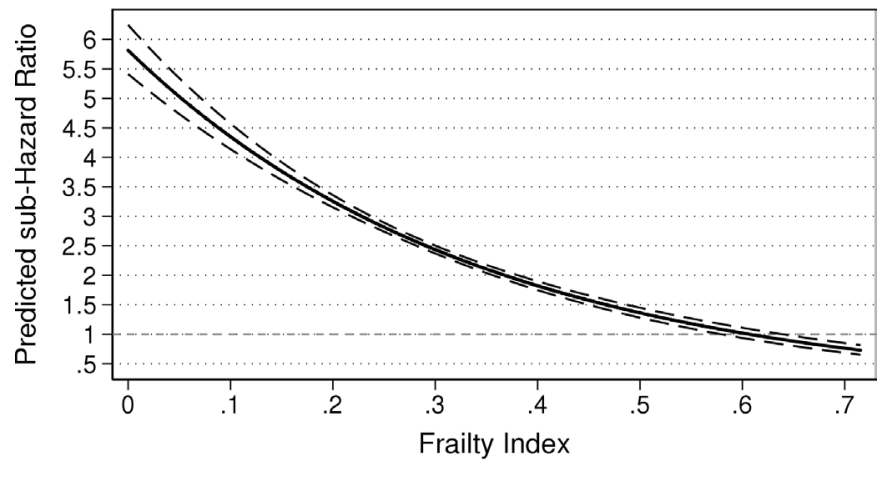

- Predicted sHR $\quad-\quad 95 \% \mathrm{Cl}$

C) Death

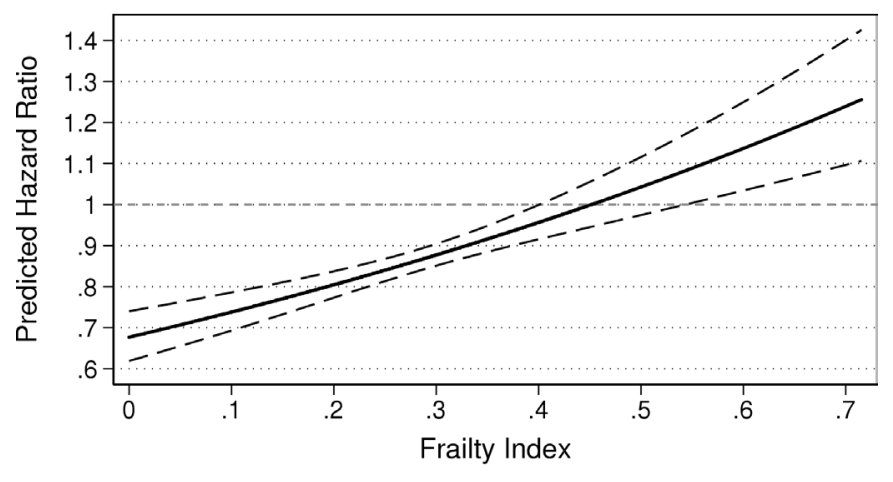

- Predicted HR - - $95 \% \mathrm{Cl}$

Figure 1 Plots of dementia-frailty (FI) interaction for 1-year health outcomes ((A) urgent hospitalisation; (B) LTC placement and (C) death), illustrating the impact of dementia (yes vs no) on outcomes across frailty (FI) level. FI, frailty index; LTC, long-term care.

competing risks, the impact of dementia on LTC admission and mortality over 1 year was significantly modified by frailty status. Specifically, the higher incidence of LTC admission and lower mortality rate evident among those with (vs without) dementia, observed overall, was attenuated with increasing frailty. There was less compelling evidence of a significant modification by client frailty for the impact of dementia on urgent hospitalisation.

Past research has shown higher healthcare utilisation (including hospitalisation and emergency department visits $^{6-10}$ for community-dwelling persons with dementia relative to controls. We found that the incidence of urgent hospitalisation, though high overall, was significantly lower among those with (vs without) dementia across all frailty levels. Our findings regarding the substantial burden of unplanned hospitalisation among community-residing older adults receiving home care, but lower incidence of hospitalisation among clients with (vs without) dementia are consistent with earlier studies of older home care recipients from North America and Europe. ${ }^{17} 18$ The lower incidence observed for clients with dementia may be explained by several factors. Our cohort included long-stay home care clients who were generally older and more impaired relative to other community-based samples. Given our primary focus on community-residing, long-stay home care clients, we also excluded clients who had received LTC or palliative care in the year prior to their index assessment. These clients would be expected to have more severe or late-stage dementia and thus, potentially different health outcomes (and drivers) compared with our study population. The coordination, monitoring and support available through home care may have contributed to the lower incidence of hospitalisation observed for clients with dementia. ${ }^{38}$ For example, persons with an explicit diagnosis of dementia receiving formal home care services may be more likely to have their unique care needs (and those of their family caregivers) identified and appropriately addressed by home care staff and other members of the interprofessional team. ${ }^{17}$ This could include a greater likelihood for such clients to have a do-not-hospitalise directive discussed and noted in their care plan. Differences in the number, type or severity of chronic conditions between the two groups may have had an effect on hospitalisation, though we adjusted for multimorbidity (and frailty) and observed no meaningful variation in incidence across frailty status. Relative to others, ${ }^{5}$ we found more similarities in the distribution of prevalent chronic conditions among clients with and without dementia. However, we also observed a significantly higher likelihood for several chronic conditions, previously shown to be important predictors of hospitalisation, ${ }^{17} 18$ among clients without (vs with) dementia, including congestive heart failure, cancer, chronic obstructive pulmonary disease and renal failure. Finally, our findings may reflect a decision not to pursue hospitalisation in more vulnerable persons with dementia. ${ }^{7}$

Consistent with the literature, both dementia and greater frailty were associated with a significantly higher incidence of LTC admission. ${ }^{11} 122125$ The attenuation of the association between dementia and incidence of LTC admission (lower sHR) with increasing frailty may initially seem counterintuitive. However, this largely reflects the important contribution of higher levels of frailty to LTC admission among clients without dementia (figure 2B). Others have similarly shown an attenuation of relative risk estimates for various health outcomes associated with dementia with increasing clinical complexity and level of comorbidity in the population under investigation. ${ }^{20}$ Our findings highlight two other important issues relevant to healthcare planning. First, dementia is a significant predictor of LTC admission among home care clients 


\section{A) Urgent Hospitalization}

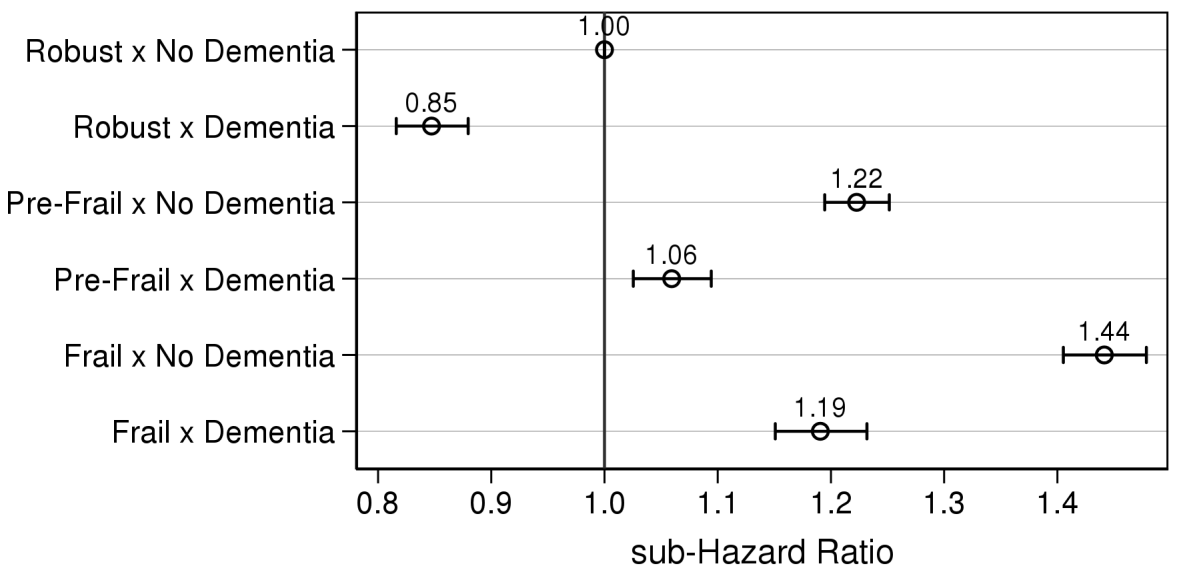

B) Long-Term Care Placement

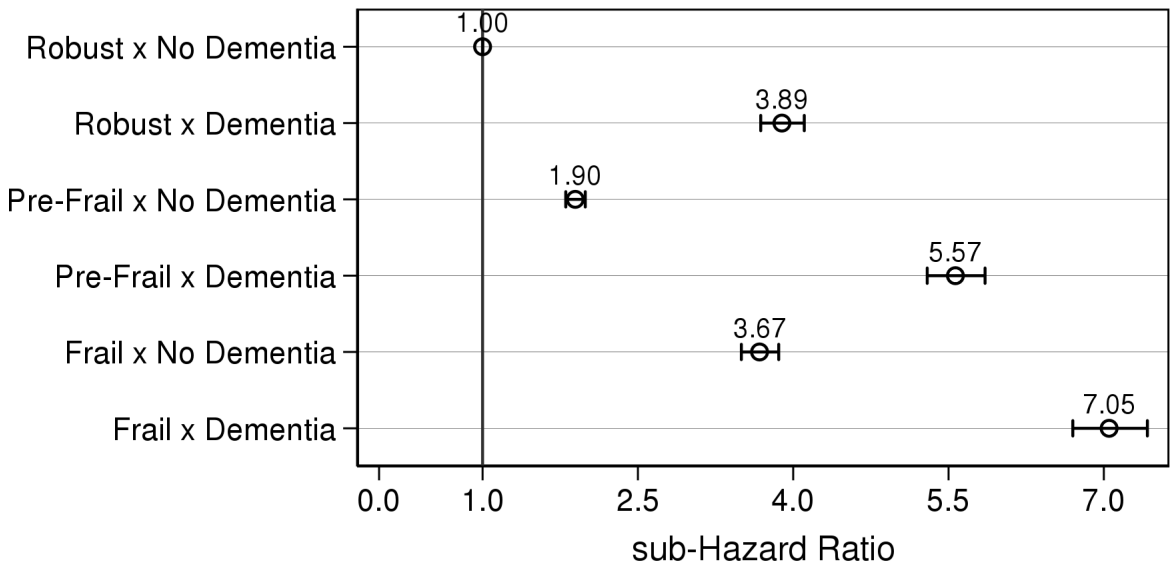

C) Death

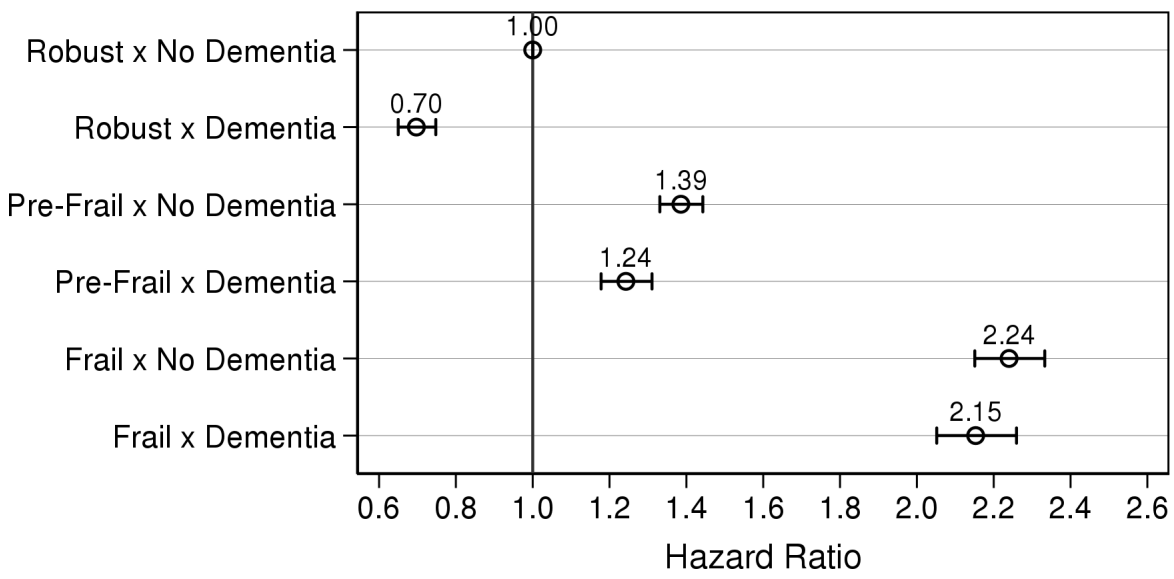

Figure 2 Plots of dementia-frailty (categorical FI) interaction for 1-year health outcomes ((A) urgent hospitalisation; (B) LTC placement and (C) death). Ratio (dementia vs no dementia) for urgent hospitalisation among robust $=0.85$; prefrail $=(1.06 / 1.22)=0.87$; frail $=(1.19 / 1.44)=0.83$. Ratio (dementia vs no dementia) for LTC admission among robust=3.89; prefrail $=(5.57 / 1.90)=2.93$; frail $=(7.05 / 3.67)=1.92$. Ratio (dementia vs no dementia) for mortality among robust $=0.70$; prefrail $=(1.24 / 1.39)=0.89$; frail $=(2.15 / 2.24)=0.96$. $\mathrm{Fl}$, frailty index; $L T C$, long-term care.

who are relatively robust (representing $34 \%$ of clients in our cohort). Second, when compared with those at lowest risk (ie, robust clients without dementia), the co-occurrence of being frail and having dementia resulted in the highest (seven-fold higher) incidence of LTC admission.
Contrary to expectations, ${ }^{12}{ }^{22}$ we observed a lower mortality rate among clients with dementia, though this association was less evident with higher levels of frailty and reversed in direction for the most frail (FI scores $\geq 0.5$ ). Though we adjusted for many factors associated with 


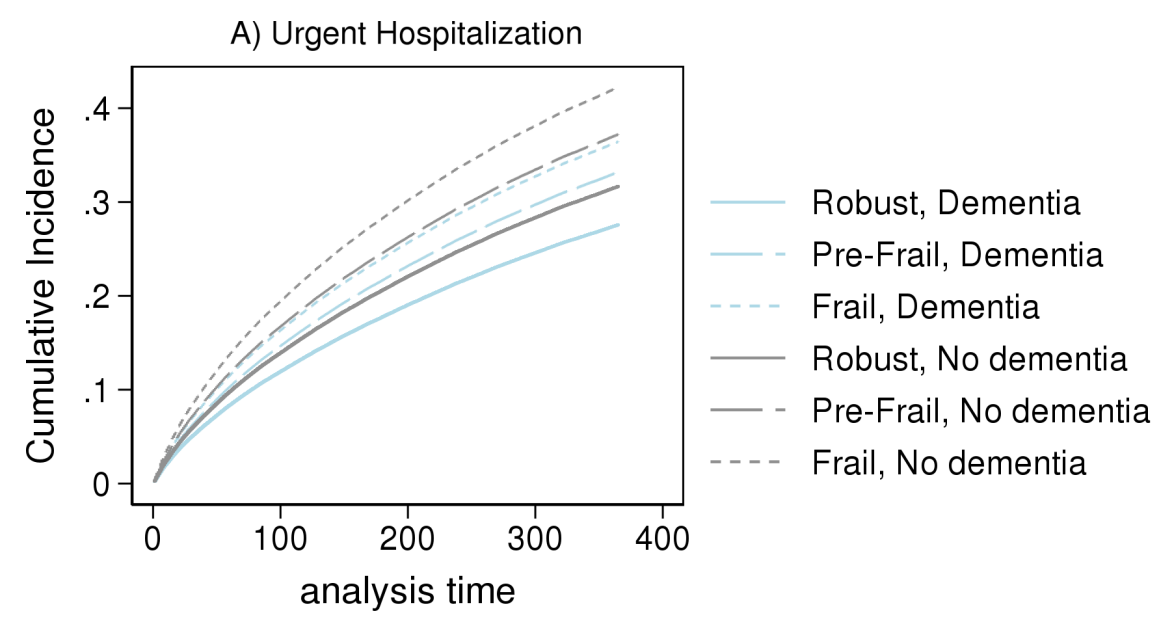

B) Long-Term Care Placement
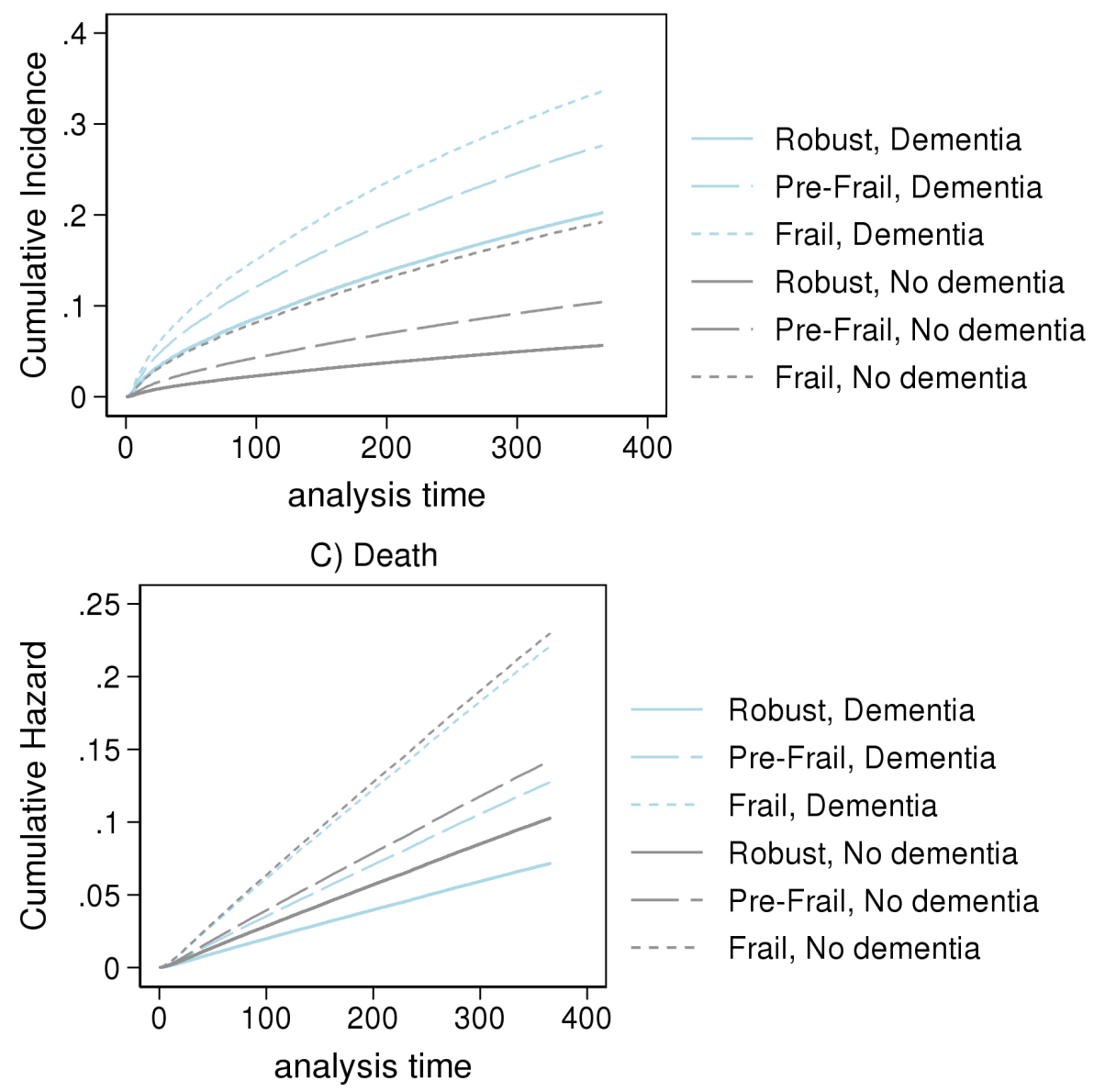

Figure 3 Plots of cumulative incidence ((A) urgent hospitalisation, (B) LTC placement) and cumulative hazard ((C) death), based on multivariable regression models that include dementia-frailty (categorical FI) interaction. FI, frailty index; LTC, long-term care.

mortality, including multimorbidity and a comprehensive frailty measure derived from physical and psychosocial items, important differences may have persisted between these two client groups, as discussed above for our hospitalisation finding. As noted earlier, it is also possible that aspects of the home care provided to clients may have resulted in better outcomes overall for those with dementia.

Strengths of our study include the population-based sample of long-stay, non-palliative clients, timeliness of data, availability of comprehensive clinical and functional measures derived from the RAI-HC and linked administrative databases and adjustment for competing risks. This allowed for a more sophisticated exploration of the joint impact of dementia and frailty on healthcare outcomes of interest to clients, healthcare practitioners and policy makers. Our analyses also employed previously validated algorithms for both dementia ${ }^{34}$ and frailty. ${ }^{24} 25$

Limitations include the absence of data for some covariates of interest (eg, the presence of advance directives, 
extent/type of supportive services), focus on all-cause outcomes, and inability to incorporate frailty as a timevarying measure. The latter issue is less of a concern given our 1-year follow-up. Our administrative data derived algorithm for dementia, though validated, ${ }^{34}$ does not allow us to comment on the relevance of dementia subtype to risk of our key outcomes, including mortality. Our findings may not be generalisable to community-residing persons with dementia or frailty not currently receiving long-stay home care or those residing in other care settings (eg, assisted living, residential or LTC) or regions with different healthcare systems. Approximately half of community-residing persons with dementia in Ontario received home care during our study period. ${ }^{39}$ Our long-stay home care population (including those with and without dementia) would be expected to be more impaired with higher multimorbidity and acuity levels than their counterparts in the community not receiving home care, ${ }^{40}$ but less functionally or cognitively impaired than similarly aged persons residing in residential or LTC facilities. ${ }^{141}$ These baseline health differences across care settings would be expected to alter the likelihood for healthcare use and outcomes among persons with and without dementia or frailty. ${ }^{1741}$ We are unable to comment on the appropriateness of patterns observed for urgent hospitalisation and LTC admission among clients with dementia and/ or frailty versus without, or on possible barriers to needed healthcare resources (eg, in rural settings). All should be areas for future dementia and frailty research.

\section{CONCLUSIONS}

Our findings support the notion that dementia and frailty, though related, represent distinct clinical considerations in our understanding of the potential impact of population ageing on healthcare utilisation and costs. ${ }^{21} 2242$ For older adults receiving home care on a long-stay basis, a population at high risk of potentially inappropriate care transitions and associated adverse outcomes, ${ }^{23}$ we showed that the likelihood for LTC admission and death (but not urgent hospitalisation) for clients with compared with those without dementia was significantly modified by their frailty status. Given projected increases in the prevalence of both dementia and frailty, ${ }^{121}$ future work should examine the extent to which the quality, appropriateness and outcomes of health and social care services vary for persons with dementia ${ }^{43}$ and with varying degrees of frailty.

\footnotetext{
Author affiliations

${ }^{1}$ School of Pharmacy, University of Waterloo, Waterlo, Ontario, Canada ${ }^{2}$ ICES, Toronto, Ontario, Canada

${ }^{3}$ Health System Performance Research Network, Toronto, Ontario, Canada ${ }^{4}$ Division of Geriatric Medicine, University of Calgary, Calgary, Alberta, Canada ${ }^{5}$ Institute of Health Policy, Management, and Evaluation, University of Toronto, Toronto, Ontario, Canada

${ }^{6}$ Division of Geriatric Psychiatry, Queen's University, Kingston, Ontario, Canada ${ }^{7}$ ICES-Queen's, Queen's University, Kingston, Ontario, Canada

${ }^{8}$ Institute for Better Health, Trillium Health Partners, Mississauga, Ontario, Canada
}

Contributors All coauthors fulfill the criteria required for authorship. CJM, LM, DBH and WPW conceived and designed the study. LM carried out the statistical analyses with assistance from MAC and CJM. LM, WPW and SEB contributed to the acquisition of relevant data. CJM wrote the manuscript and all authors (LM, DBH, MAC, SEB, DPS and WPW) contributed substantially to the critical appraisal, review and interpretation of findings and the final preparation of the manuscript. All authors claim responsibility for the integrity of the data and analyses and have approved the submitted version of the manuscript.

Funding This research was supported by a grant from the Ontario Ministry of Health and LongTerm Care (MOHLTC) to the Health System Performance Research Network (HSPRN: fund \#06034, recipient WPW), and by ICES, which is also funded by an annual grant from the Ontario MOHLTC. The opinions, results and conclusions reported in this paper are those of the authors and are independent from funding sources. No endorsement by ICES or the Ontario MOHLTC is intended or should be inferred. Parts of this material are based on data and information compiled and provided by the Canadian Institute for Health Information (CIHI). However, the analyses, conclusions, opinions and statements expressed herein are those of the authors, and not necessarily those of $\mathrm{CIHI}$. We thank IMS Brogan for use of their Drug Information Database.

Competing interests DPS has participated as a site investigator for a clinical trial sponsored by Hoffman La Roche.

Patient consent for publication Not required.

Provenance and peer review Not commissioned; externally peer reviewed.

Data sharing statement The data from this study are held securely in coded form at ICES. While data sharing agreements prohibit ICES from making the data publicly available, access may be granted to those who meet pre-specified criteria for confidential access, available at www.ices.on.ca/DAS.

Open access This is an open access article distributed in accordance with the Creative Commons Attribution Non Commercial (CC BY-NC 4.0) license, which permits others to distribute, remix, adapt, build upon this work non-commercially, and license their derivative works on different terms, provided the original work is properly cited, appropriate credit is given, any changes made indicated, and the use is non-commercial. See: http://creativecommons.org/licenses/by-nc/4.0/.

\section{REFERENCES}

1. Chambers LW, Bancej C, McDowell I, (eds). Prevalence and Monetary Costs of Dementia in Canada. Toronto (ON): Alzheimer Society of Canada, 2016. Available: http://alzheimer.ca/sites/default/ files/Files/national/Statistics/PrevalenceandCostsofDementia_EN.pdf [Accessed 8 Oct 2018].

2. Maxwell CJ, Campitelli MA, Diong C, et al. Variation in the health outcomes associated with frailty among home care clients: relevance of caregiver distress and client sex. BMC Geriatr 2018;18:211.

3. Mondor L, Maxwell CJ, Hogan DB, et al. Multimorbidity and healthcare utilization among home care clients with dementia in Ontario, Canada: A retrospective analysis of a population-based cohort. PLoS Med 2017;14:e1002249.

4. Ng R, Maxwell CJ, Yates EA, et al. Brain disorders in Ontario: prevalence, incidence and costs from health administrative data. Toronto: Institute for Clinical Evaluative Sciences, 2015. Available: http://www.ices.on.ca/ /media/Files/Atlases-Reports/2015/BrainDisorders-in-Ontario/Full-Report.ashx [Accessed 14 Apr 2019].

5. Frytak JR, Henk HJ, Zhao Y, et al. Health service utilization among Alzheimer's disease patients: evidence from managed care. Alzheimers Dement 2008;4:361-7.

6. Lin PJ, Fillit HM, Cohen JT, et al. Potentially avoidable hospitalizations among Medicare beneficiaries with Alzheimer's disease and related disorders. Alzheimers Dement 2013;9:30-8.

7. Feng Z, Coots LA, Kaganova Y, et al. Hospital and ED use among Medicare beneficiaries with dementia varies by setting and proximity to death. Health Aff 2014;33:683-90.

8. Phelan EA, Borson S, Grothaus L, et al. Association of incident dementia with hospitalizations. JAMA 2012;307:165-72.

9. Zhao Y, Kuo TC, Weir S, et al. Healthcare costs and utilization for Medicare beneficiaries with Alzheimer's. BMC Health Serv Res 2008;8:108.

10. Bynum JP, Rabins PV, Weller W, et al. The relationship between a dementia diagnosis, chronic illness, medicare expenditures, and hospital use. J Am Geriatr Soc 2004;52:187-94.

11. Callahan CM, Arling G, Tu W, et al. Transitions in care for older adults with and without dementia. J Am Geriatr Soc 2012;60:813-20. 
12. Callahan $\mathrm{CM}, \mathrm{Tu} \mathrm{W}$, Unroe $\mathrm{KT}$, et al. Transitions in care in a nationally representative sample of older Americans with dementia. J Am Geriatr Soc 2015;63:1495-502.

13. Tropea J, LoGiudice D, Liew D, et al. Poorer outcomes and greater healthcare costs for hospitalised older people with dementia and delirium: a retrospective cohort study. Int $J$ Geriatr Psychiatry 2017;32:539-47.

14. Zilberberg MD, Tjia J. Growth in dementia-associated hospitalizations among the oldest old in the United States: implications for ethical health services planning. Arch Intern Med 2011;171:1850-1.

15. Expert Group on Home \& Community Care. Report of the Expert Group on Home \& Community Care. Bringing Care Home. 2015. Available: http://health.gov.on.ca/en/public/programs/ccac/docs/ hcc report.pdf [Accessed 9 Apr 2019].

16. Institute of Medicine (IOM) and National Research Council (NRC). The future of home health care: Workshop summary. Washington, DC: The National Academies Press, 2015. Available: http:// nationalacademies.org/hmd/reports/2015/future-home-health-care. aspx [Accessed 9 Apr 2019].

17. Rönneikkö JK, Mäkelä M, Jämsen ER, et al. Predictors for unplanned hospitalization of new home care clients. J Am Geriatr Soc 2017:65:407-14.

18. Morris JN, Howard EP, Steel K, et al. Predicting risk of hospital and emergency department use for home care elderly persons through a secondary analysis of cross-national data. BMC Health Serv Res 2014;14:519.

19. Health Quality Ontario. The Reality of Caring: Distress among the caregivers of home care patients. Toronto: Queen's Printer for Ontario, 2016. Available: http://www.hqontario.ca/Portals/ 0/documents/system-performance/reality-caring-report-en.pdf [Accessed 9 Apr 2019].

20. Tonelli M, Wiebe N, Straus S, et al. Multimorbidity, dementia and health care in older people:a population-based cohort study. CMAJ Open 2017;5:E623-E631.

21. Liu Z, Han L, Gahbauer EA, et al. Joint trajectories of cognition and frailty and associated burden of patient-reported outcomes. J Am Med Dir Assoc 2018;19:304-9.

22. St John PD, Tyas SL, Griffith LE, et al. The cumulative effect of frailty and cognition on mortality - results of a prospective cohort study. Int Psychogeriatr 2017;29:535-43.

23. Bergman H, Ferrucci L, Guralnik J, et al. Frailty: an emerging research and clinical paradigm-issues and controversies. $J$ Gerontol A Biol Sci Med Sci 2007;62:731-7.

24. Campitelli MA, Bronskill SE, Hogan DB, et al. The prevalence and health consequences of frailty in a population-based older home care cohort: a comparison of different measures. BMC Geriatr 2016;16:133.

25. Hogan DB, Freiheit EA, Strain LA, et al. Comparing frailty measures in their ability to predict adverse outcome among older residents of assisted living. BMC Geriatr 2012;12:56.

26. Haaksma ML, Rizzuto D, Ramakers I, et al. The impact of frailty and comorbidity on institutionalization and mortality in persons with dementia: a prospective cohort study. J Am Med Dir Assoc 2019;20.

27. Kelaiditi E, Andrieu S, Cantet C, et al. Frailty index and incident mortality, hospitalization, and institutionalization in Alzheimer's disease: data from the ICTUS Study. J Gerontol A Biol Sci Med Sci 2016;71:543-8.
28. Feng L, Nyunt MS, Gao Q, et al. Physical frailty, cognitive impairment, and the risk of neurocognitive disorder in the Singapore Longitudinal Ageing Studies. J Gerontol A Biol Sci Med Sci 2017;72:369-75.

29. Gross AL, Xue QL, Bandeen-Roche K, et al. Declines and impairment in executive function predict onset of physical frailty. $J$ Gerontol $A$ Biol Sci Med Sci 2016;71:1624-30.

30. Chow S, Chow R, Wan A, et al. National Dementia Strategies: What Should Canada Learn? Can Geriatr J 2018;21:173-209.

31. Health Quality Ontario. Measuring Up 2018: A yearly report on how Ontario's health system is performing. Toronto, ON: Queen's Printer for Ontario, 2018. Available: https://www.hqontario.ca/SystemPerformance/Yearly-Reports/Measuring-Up-2018 [Accessed 9 Apr 2019].

32. Canadian Institute for Health Information. Data Quality Documentation, Home Care Reporting System, 2012-2013. Ottawa, ON: CIHI, 2013. Available: https://secure.cihi.ca/free_products/ HCRS-External-Data-Quality-Report_2012_EN_web.pdf [Accessed 8 Oct 2018].

33. Health Quality Ontario. Palliative Care at the End of Life. Toronto: Queen's Printer for Ontario, 2016. Available: https://www.hqontario. $\mathrm{ca} /$ Portals/0/documents/system-performance/palliative-care-reporten.pdf

34. Jaakkimainen RL, Bronskill SE, Tierney MC, et al. Identification of physician-diagnosed Alzheimer's disease and related dementias in population-based administrative data: a validation study using family physicians' electronic medical records. J Alzheimers Dis 2016:54:337-49.

35. Theou O, Tan EC, Bell JS, et al. Frailty levels in residential aged care facilities measured using the frailty index and FRAIL-NH Scale. J Am Geriatr Soc 2016;64:e207-e212.

36. Pefoyo AJ, Bronskill SE, Gruneir A, et al. The increasing burden and complexity of multimorbidity. BMC Public Health 2015;15:415.

37. Austin PC, Fine JP. Practical recommendations for reporting Fine-Gray model analyses for competing risk data. Stat Med 2017;36:4391-400.

38. Ju YJ, Lee HJ, Kim W, et al. Association between home-visit nursing utilization and all-cause hospitalization among long-term care insurance beneficiaries: A retrospective cohort study. Int J Nurs Stud 2017:75:93-100.

39. Health Quality Ontario. Why we need a quality standard for dementia care in the community. Toronto, ON: Queen's Printer for Ontario, 2018. Available. https://www.hqontario.ca/portals/0/documents/ evidence/quality-standards/qs-dementia-infographic-en.pdf. (Accessed 14 Apr 2019).

40. Bronskill SE, Corbett L, Gruneir A. Introduction. In: Bronskill SE, Camacho X, Gruneir A, MM H, et al. eds. Health System Use by Frail Ontario Seniors: An In-Depth Examination of Four Vulnerable Cohorts. Toronto, ON: Institute for Clinical Evaluative Sciences, 2011.

41. Maxwell CJ, Amuah JE, Hogan DB, et al. Elevated Hospitalization Risk of Assisted Living Residents With Dementia in Alberta, Canada. J Am Med Dir Assoc 2015;16:568-77.

42. Mondor L, Maxwell CJ, Hogan DB, et al. The Incremental Health Care Costs of Frailty Among Home Care Recipients With and Without Dementia in Ontario, Canada: A Cohort Study. Med Care 2019:57:512-20.

43. Warrick N, Prorok JC, Seitz D. Care of community-dwelling older adults with dementia and their caregivers. CMAJ 2018;190:E79 4-E799. 